\title{
Numerical Investigations of Rack Storage Fires
}

\author{
Zhenghua Yan and Göran Holmstedt \\ Department of Fire Safety Engineering, Lund University, Box 118, S-221 00, Sweden
}

\begin{abstract}
A number of numerical simulations of rack storage fires have been carried out, with various fuel types and burner outputs. Both the standard buoyancy-modified $k-\varepsilon$ turbulence model and a recently developed turbulence model which significantly improves the consideration of the buoyancy effect on turbulence and turbulent transport, were used to study the turbulence of the buoyant flow. The flamelet concept, coupled to a prescribed probability density function, was employed to model the non-premixed combustion process. Sooting was modeled by solving the balance equations for mass fraction and number density considering nucleation, surface growth, coagulation and oxidation. The discrete transfer method was used to calculate radiation, with the radiation properties of the main radiating species - carbon dioxide, water vapour and soot, provided by a fast, narrowband model. The results, including heat flux and gas temperature profile, were analyzed and compared with experimental measurements. The comparisons showed considerably improved agreement for the new model.
\end{abstract}

KEYWORDS: CFD, Turbulence models, Heat transfer, Soot formation, Rack fires

\section{INTRODUCTION}

In warehouses, a large amount of goods is usually stored in racks. Rack storage has the advantage of ease of access. However, the potential fire hazard in rack storage is high due to the large surface area of combustible material. The study of rack storage fires is thus of great importance. 
Recently, Ingason and de Ris [1] carried out a series of experımental studies on rack storage fires, varying the fuel type, fire power and rack separation distance. They measured the heat flux, gas temperature and gas velocity at various locations. Based on their experimental data, they also provided an empirical formula for the estimation of the heat flux. However, due to the complexity of the problem, the validity of their empirical correlation is limited.

As an effective and more fundamentally based tool, CFD (Computational Fluid Dynamics) can provide a comprehensive description on the physics in fires, and thus plays an important role in fire research. With the rapid expansion of computer capacity and progress being made in sub-models, CFD is becoming more popular and more important in both research and applications.

In many practical CFD computations, turbulence still imposes a major difficulty at present, although great effort has been made in the history to pursue a strategy to consider turbulence, which is unfortunately a property of flow, in such a way that can be as economic and universal as possible. For a turbulent flow, different solution strategies can be adopted, including the turbulence modeling or Reynolds Averaged Simulation (RAS), Large Eddy Simulation (LES) and Direct Numerical Simulation (DNS), each having its own advantages and disadvantages with regard to the simplicity, universality and efficiency.

In this study, a number of three-dimensional numerical simulations for rack storage fires were made, based on the turbulence modeling strategy, where the instantaneous equations are statistically averaged for solution and the contribution of fluctuation to the averaged values is modeled using models. By solving a set of coupled Favre-averaged governing equations, the fluid dynamics, turbulent combustion, sooting, radiation, the interaction between the wall and the gas, and the heat conduction inside the wall were all considered. Both the standard buoyancy modified $k-\varepsilon$ turbulence model and a recently developed turbulence model were used for turbulence modeling and compared. The results, including heat flux and gas temperature profile, were analyzed and compared with experimental measurements [1].

\section{THEORETICAL MODELS}

The physical processes involved in fires are assumed to be described by a set of theoretical equations and models. These equations are numerically solved to provide insight into the simulated fire. In this study, continuity, momentum, energy, species, radiation and state equations are solved, with turbulence, turbulent combustion, soot and radiation models employed.

Since fire is usually buoyancy dominated, with a Froude number below 1.0, the effect of buoyancy on turbulence and turbulent transport is important. It has been found in many earlier studies [2-4], that the standard buoyancy-modified $k-\varepsilon$ model does not take this buoyancy effect into account properly and tends to seriously under-predict the spreading 
rate of a buoyant plume. A recently developed new turbulence model, which was found to be promising, economic and stable [4], was implemented to improve the consideration of the buoyancy effect. In this new turbulence model, improvement was made to consider more properly the turbulent transport of scalars and the contribution of buoyancy to the production of turbulence kinetic energy.

The flamelet concept [5-7] was employed to model the non-premixed combustion process, with a prescribed probability density function (pdf) assumed to be in the form of a normalized Beta function.

A two-variable soot model proposed by Lindstedt [8,9] was adopted to consider soot, which can be important in radiation calculations. In this soot model, soot formation is related to the chemical intermediate acetylene, rather than to the parent fuel, which was used in the soot model suggested by Moss et al. [10].

The radiation equation was solved using the Discrete Transfer (DT) method [11], which provides a good compromise between accuracy and economy. The radiation properties of the combustion products are highly non-grey, and were thus calculated using a fast narrowband model - FASTNB [12], which is based on spectral calculation and predicts the radiation intensity in a generally non-isothermal and non-homogeneous combustion environment. The solid surfaces were assumed to be diffuse and grey.

Since the interaction between the gas and solid phases is important, the heat conduction inside the solid boundaries was assumed to be one-dimensional and was taken into account by solving the corresponding heat conduction equation.

The details of the theoretical models can be found in $[4,12,13]$.

\section{BRIEF DESCRIPTION OF THE PROBLEM}

The experiments were carried out by Ingasson and de Ris [1]. As shown in Fig. 1, a gas burner, $60 \mathrm{~mm}$ in diameter, is located at the center of the gap between four towers. These towers were used to represent the storage racks. The tower sides facing the flame were constructed of $5 \mathrm{~mm}$ thick steel sheet (carbon). The vertical distribution of the heat flux was measured at the measurement point shown in Fig. 1. Ideally, the problem is symmetric. Thus, the computation was performed on a quarter of the experimental domain with only one tower included, using symmetrical boundary conditions. However, in experiment, since the turbulent flow generated by buoyancy is quite sensitive to external influences, the flame was observed to lean away from the axis of symmetry, although great effort was made to keep the flame as symmetric as possible [1]. Each experiment lasted for 150 seconds and the experimental heat flux given is an average over a period of 50 seconds.

The thermal properties of the steel were taken to be: $\rho=7800 \mathrm{~kg} / \mathrm{m}^{3}, c_{p}=460 \mathrm{~J} / \mathrm{kgK}$. $\lambda=73.0-0.043 \times T \mathrm{~W} / \mathrm{mK}$ for $300 \mathrm{~K} \leq T \leq 1000 \mathrm{~K}$, where $T$ is the steel temperature in 
$K[1,14]$. Since the emissivity of a metal surface can vary significantly with its physical state, the emissivity of this steel, which was exposed to a flame, was assumed to be 0.9 .

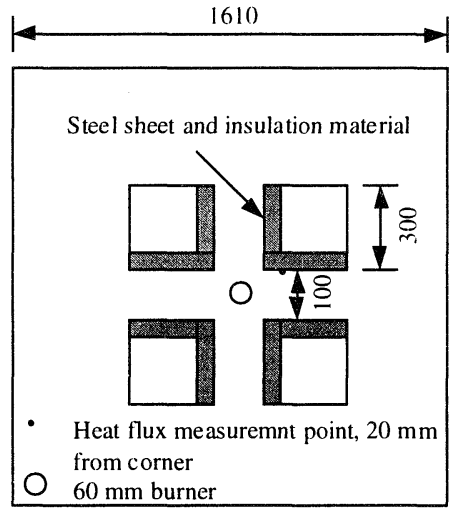

Unit: mm

A) Plan view

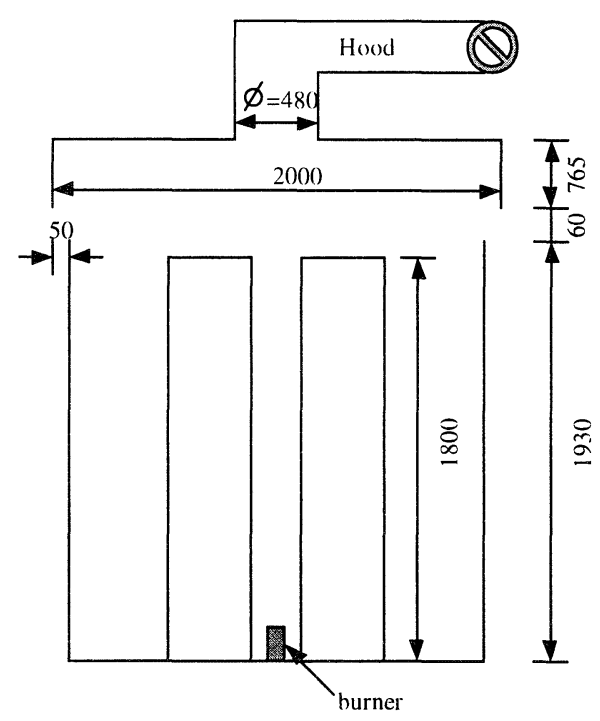

B) Side view

FIGURE 1 Configuration of the simulated experiment

\section{NUMERICAL CONSIDERATION AND SENSITIVITY ANALYSIS}

As indicated by the simulation results shown in Fig. 2, the gas phase reaches a virtually steady state quickly, due to its small inertia. In contrast, due to its large inertia, the heat transfer process inside the solid materials is still transient during the period considered. The wall surface temperature increases almost linearly with time. However. the rate of wall temperature increase is low, and it has little effect on the gas phase and the flame heat transfer. Thus, the local heat flux imposed on the wall boundary also reaches a virtually constant value shortly after ignition as shown in Fig. 2. Therefore, the computation was performed for only the first 60 seconds of the experimental period.

The computations were carried out using non-uniform grid. Before performing the final calculations, the sensitivity of the results to the grid refinement, DT ray number and time step was analyzed. The sensitivity analysis calculations were performed for a typical case 
for a period of 30 seconds, which was deemed to be sufficient. The results presented below are practically time step independent, grid-independent and ray number-independent.

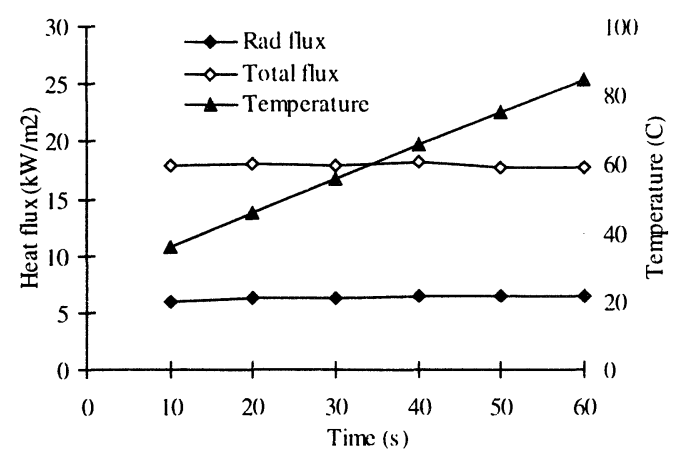

FIGURE 2 Variation of steel surface temperature and heat flux with time at a point $20 \mathrm{~mm}$ from the corner and $1.2 \mathrm{~m}$ from the floor

\section{RESULTS AND DISCUSSION}

The simulation was carried out for four different cases, with various fuels and burner outputs. These four cases are: Case 1: propane, $70.4 \mathrm{~kW}$; Case 2: propane, $44.3 \mathrm{~kW}$; Case 3: carbon monoxide, $60.8 \mathrm{~kW}$; Case 4: propane, $118 \mathrm{~kW}$. In order to show the significant effect of turbulence modeling on the simulations, both a recently developed turbulence model [4] and the standard buoyancy-modified $k-\varepsilon$ model [15] were used. For brevity, we will henceforth denote these two models the new model and the standard model. Since the gas phase properties and the local heat flux imposed on the wall become almost constant shortly after ignition, we used the predicted gas phase and heat flux data at time $=60$ seconds after ignition for the analysis.

Figures 3-6 show a comparison between the computed and the measured vertical distribution of total heat flux along a line $20 \mathrm{~mm}$ away from the tower corner. As indicated by these figures, the agreement between experiment and the prediction using the new model is reasonably good, and much better than that between experiment and the prediction using the standard model. When the new model was used, the general variation in the heat flux was reproduced by the computation and there is good agreement between the locations of the measured and predicted peak values in all four cases studied. However, since the standard model tends to seriously under-predict the entrainment of a buoyant flow and thus over-predict the length of a buoyant diffusion flame [2-4], the peak value locations in all four cases were consistently shifted to a much higher location when the standard model was adopted. This analysis confirms that it is of critical importance to replace or improve the standard model in the future fire simulations. 
Some significant differences can be seen in Figs. 3-6. It can be easily seen that in all four cases, the rate of decrease in the predicted heat flux in the post-flame zone is much lower than that measured. The plume temperature is likely to be the major source of this difference. As will be discussed later, the measured plume temperature decreases much faster with height than that predicted. The uncertainty in the convection heat transfer computation could also be responsible for this discrepancy. In this study, the standard wall function was used to compute the convection heat transfer, due to limitation of computer resources. The uncertainty thus introduced could be reduced by refining the computation grid and considering low-Reynolds-number effects on turbulence modeling in future work. Unfortunately, due to the complexity of the problem and the experimental uncertainty, it is very difficult to quantify the theoretical uncertainties in the present study.

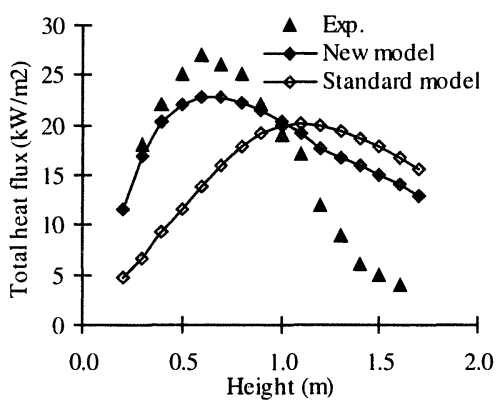

FIGURE 3 Heat flux comparison, Case 1

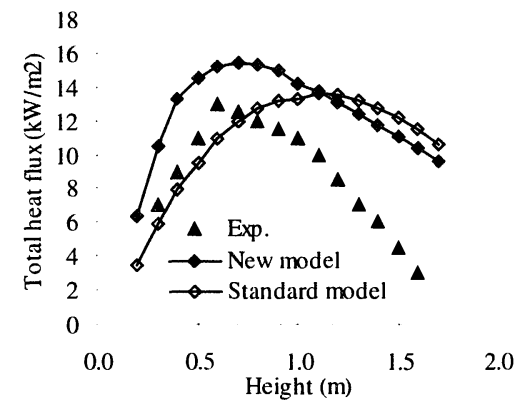

FIGURE 5 Heat flux comparison, Case 3

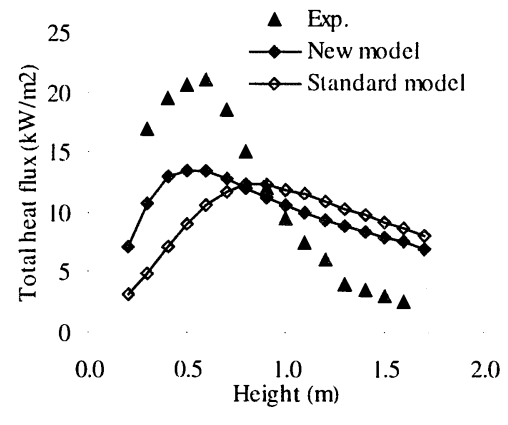

FIGURE 4 Heat flux comparison, Case 2

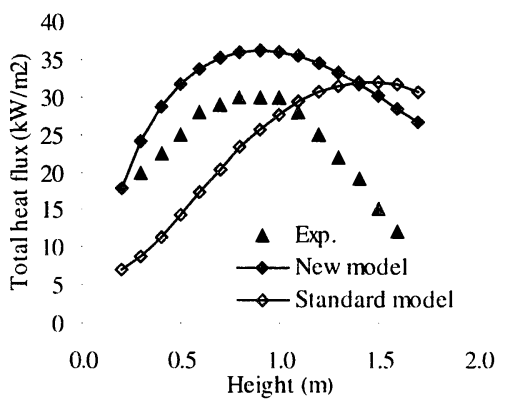

FIGURE 6 Heat flux comparison, Case 4

Another major deficiency is that in Case 2, the total heat flux at low locations was significantly under-predicted, even when the new model was employed. The peak value 
was under-predicted by about $40 \%$. In this case, the burner had an output such that the flame was just wide enough to attack the tower surface. Since the temperature gradient at the flame edge is very high, a small difference in the flame width would result in a significant change in convection heat transfer. Thus, the heat transfer is very sensitive to the flame width in this case.

Figure 7 shows a comparison of computed radiation fluxes in two different cases: Case I and Case 3. It shows that the radiation flux in Case 1 is almost twice that in Case 3. This may largely be attributed to soot radiation. In Case 1, the fuel is propane and soot is formed. But there is no soot in Case 3 where the fuel is carbon monoxide. Since the carbon monoxide flame temperature is likely to be a little higher than that of propane, and the burner output in Case 1 is only about $15 \%$ higher than that in Case 3, the higher radiation flux in Case 1 is likely to be mainly due to soot radiation. However, since the flame is not big and a propane flame is not very sooty, it can be seen, by comparing Fig. 3 with Fig. 7. that the total heat flux is not yet dominated by radiation.

Figure 8 shows comparison between measured and predicted steel temperature histories at two locations in Case 1. Both experiment and theoretical calculation indicate that the steel temperature increases linearly with time. This implies that the imposed heat flux at each point is essentially constant. The good agreement observed in this figure is a direct result of the good agreement between the predicted and measured heat fluxes at these two points, as shown Fig. 3.

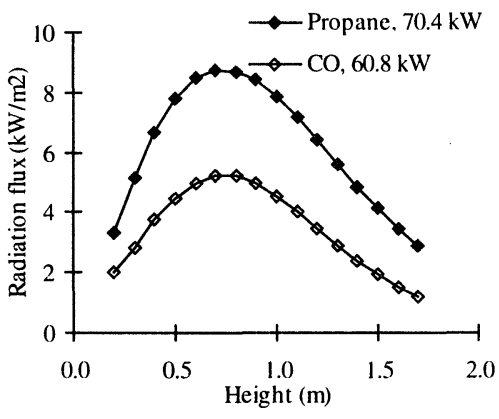

FIGURE 7 Computed radiation flux for different fuels, new model used

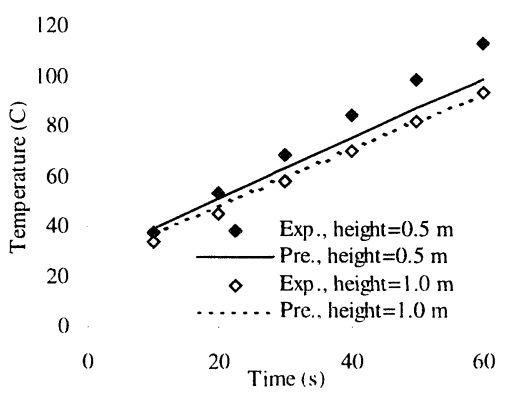

FIGURE 8 Comparison of measured and predicted steel temperature at two points which are $20 \mathrm{~mm}$ away from tower corner and at two heights (Case 1 , new turbulence model used) 
In experiments, gas temperature and velocity profiles were measured using sheathed 0.25 $\mathrm{mm}$ type $\mathrm{K}$ thermocouples and bi-directional probes. No radiation effect on the thermocouples was considered. Fig. 9 shows the comparison of horizontal gas temperature profile at the tower gap center at a height of $1.07 \mathrm{~m}$ above the floor in Case 1. Since this height was predicted to be below the flame tip, both models gave essentially the same central line temperature. It can be seen that, although the new model gives a greater plume width than the standard model, the measured plume width is much greater than that predicted by either of the models. Since no radiation effect on the thermocouples was considered, the measured temperatures at the plume center and edge are likely to be somewhat lower and higher, respectively, than the real values. For comparison, extra computations were carried out to simulate the corresponding free flame case when the towers were removed. The corresponding computed horizontal gas temperature profiles are shown in Fig. 10. The decay of the central line temperature is compared in Fig. 11 with that given by Heskestad's empirical formula [16], which is well accepted. The results shown in Figs. 10 and 11 indicate a significant improvement on entrainment with the new turbulence model. Upon comparing Fig. 9 with Fig. 10, it can be seen that the theoretical predictions indicate that the entrainment was significantly blocked when the towers were present, but no obvious blocking effect on the entrainment by the towers can be seen in the measurements. Intuitively, it is reasonable to expect a significant blocking effect on the entrainment by the towers. However, in the experiments, the fact that the flame fluttered and leaned away from the symmetry axis [1] may compensate for the block effecting on the entrainment by the towers. Therefore, the large gas temperature difference between the predictions and the measurements is thought to be significantly attributable to the uncertainties in both the experimental control and the gas phase measurements.

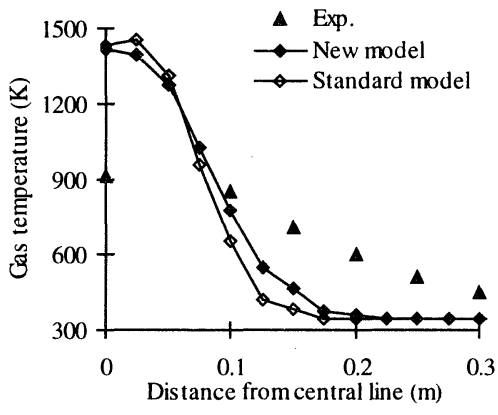

FIGURE. 9 Comparison of gas temperature profile, Case 1. Note: Exp. indicates data averaged over a oneminute period , 60-120 seconds after ignition (from Haukur Ingason's original data)

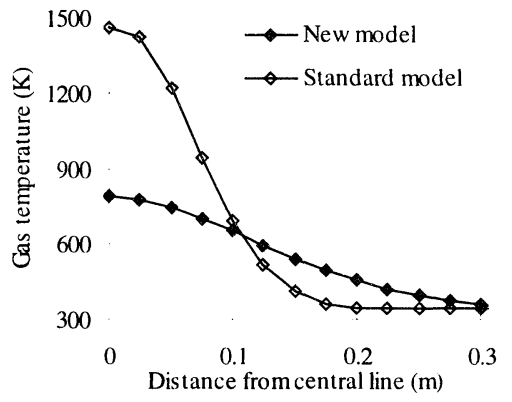

FIGURE. 10 Computed gas temperature profile for a free flame corresponding to Case 1 


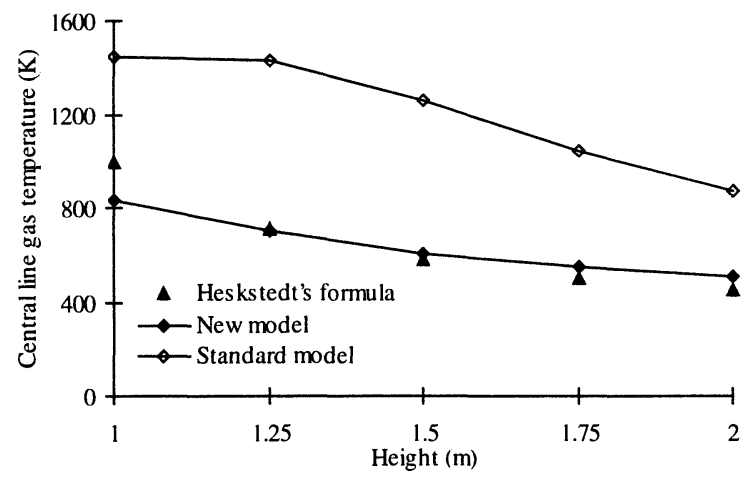

FIGURE 11 Decay of central line gas temperature for a free flame corresponding to Case 1

\section{CONCLUSIONS}

Rack storage fires have been numerically investigated using the CFD methodology. The computed heat flux and gas temperature were analyzed and compared with experimental measurements. Both a recently developed turbulence model and the standard buoyancymodified $k-\varepsilon$ turbulence model were used in the computations. The agreement between prediction and measurement was considerably improved when the new model was used. It can be concluded that the turbulence modeling is important in the prediction of the plume entrainment and the shape of a buoyant diffusion flame. It is essential to replace or improve the currently widely used standard turbulence model in fire simulations.

Some significant differences were observed between measurement and the prediction given by both the standard buoyancy modified $k-\varepsilon$ model and the new model which was found to work well for free flames and plumes. However, due to the complexity of the problem and the uncertainties involved in the experiments, no specific aspect of theoretical uncertainties could be quantified. In order to pinpoint the reasons for the discrepancy, it would be necessary to clarify the specific effect of the walls on turbulence in this particular configuration. A detailed accurate measurement, or Direct Numerical Simulation, of an inert thermal plume in a closely corresponding geometry, can be helpful on this aspect.

\section{ACKNOWLEDGEMENT}

This work was supported by the Swedish Fire Research Board (BRANDFORSK) and the Center for Combustion Science and Technology (Sweden), who are gratefully 
acknowledged. The authors also wish to express special thanks to Dr Haukur Ingasson (SP, Sweden) for his original data.

\section{REFERENCES}

1. Ingason, H. And de Ris, J., "Flame Heat Transfer in Storage Geometries", submitted to Fire Safety Journal, 1996

2. Annarumma, M. O., Most, J. M. and Joulain, P., "On the Numerical Modeling of Buoyancy-Dominated Turbulent vertical Diffusion Flames", Combustion and Flame, $85: 403-415,1991$

3. Nam, S. and Bill R. G., "Numerical Simulation of Thermal Plumes", Fire Safety Journal, 21:231-256, 1993

4. Yan, Z. H. and Holmstedt, G., "A Two-Equation Turbulence Model and Its Application to a Buoyant Diffusion Flame", International Journal of Heat and Mass Transfer, 42:1305-1315. 1999

5. Peters, N., "Laminar Flamelet Concepts in Turbulent Combustion", Twenty-first Symp. (International) on Combustion, The Combustion Institute, Pittsburgh, p. 12311250,1986

6. Peters, N., "Laminar Diffusion Flamelet Model in Non-premixed Turbulent Combustion”, Prog. Energy Combust. Sci., 10:319-339, 1984

7. Liew, S. K., Bray, K. N. C., and Moss, J. B., "A Stretch Laminar Flamelet Model of Turbulent Nonpremixed Combustion", Combustion and Flame, 56:199-213, 1984

8. Lindstedt, R. P., "A Simple Reaction Mechanism for Soot Formation in Non-premixed Flames", IUTAM Conference on Aerothermo-chemistry in Combustors, Taiwan, 1991

9. Fairweather, M., Jones, W. P., Ledin, H. S., and Lindstedt, R. P., "Prediction of Soot Formation in Turbulent Non-premixed Propane Flames", Twenty-Fourth Symposium (International) on Combustion, The Combustion Institute, Pittsburgh, p. 1067-1074. 1992

10. Moss, J. B., Stewart, C. D., and Syed, K. J. "Flowfield Modeling of Soot Formation at Eleveted Pressure", Twenty-Second Symposium (International) on Combustion, The Combustion Institute, Pittsburgh, p. 413-423, 1988

11. Lockwood F. C. \& Shah N. G., "A New Radiation Solution Method for Incorporation in General Combustion Prediction Procedures", Eighteenth Symposium (International) on Combustion, The Combustion Institute, Pittsburgh, p. 1405-1414, 1981 
12. Yan, Z. H., and Holmstedt, G., "Fast, Narrow-band Computer Model for Radiation Calculations", Numerical Heat Transfer, Part B: Fundamentals, 31:61-71, 1997

13. Yan, Z. H., and Holmstedt, G., "Three-dimensional Computation of Heat Transfer from Flames Between Vertical Parallel Walls", Combustion and Flame, 117:574-588, 1999

14. Liley, P. E., "Steam Tables in SI Units, private communication", School of Mechanical Engineering, Purdue University, 1978

15. Rodi, W., "Turbulence Models and Their Application in Hydraulics - A State of The Art Review", SBF Report 80/T/125, University of Karlsruhe, 1980

16. Heskestad, G., "Engineering Relations for Fire Plume”, Fire Safety Journal, 7:25-32, 1984 
